\title{
Peculiarities in the orbital and precessional variability of SS433 from INTEGRAL observations
}

\section{A. Cherepashchuk ${ }^{* a}$, R. Sunyaev ${ }^{b}$, S. Molkov ${ }^{b}$, E. Antokhina ${ }^{a}$, K. Postnov ${ }^{a}$, A. Bogomazov $^{a}$}

${ }^{a}$ Moscow M.V. Lomonosov State University, Sternberg Astronomical Institute, 119992 Moscow, Russia

${ }^{b}$ Space Research Institute of Russian Academy of Sciences, Moscow, Russia

E-mail: cherepashchuk@gmail.com, elant@sai.msu.ru,

kpostnov@gmail.com, molkov@hea.iki.rssi.ru

\begin{abstract}
Based on multiyear INTEGRAL observations of SS433, a composite IBIS/ISGRI 18-60 keV orbital light curve is constructed around zero precessional phase $\psi_{p r}=0$, which corresponds to a maximum separation of the moving emission lines originated in sub-relativistic jets from the source. It shows a peculiar shape characterized by a significant excess near the orbital phase $\phi_{\text {orb }}=0.25$, which is not seen in the softer $2-10 \mathrm{keV}$ energy band. Such a shape is likely to be due to a complex asymmetric structure of the funnel in a supercritical accretion disk in SS433. The orbital light curve at 40-60 keV demonstrates two almost equal bumps at phases $\sim 0.25$ and $\sim 0.75$, most likely due to nutation effects of the accretion disk. The change of the offeclipse 18-60 keV X-ray flux with the precessional phase shows a double-wave form with strong primary maximum at $\psi_{p r}=0$ and weak but significant secondary maximum at $\psi_{p r}=0.6$. A weak variability of the $18-60 \mathrm{keV}$ flux in the middle of the orbital eclipse correlated with the disk precessional phase is also observed. The joint analysis of the broadband (18-60 keV) orbital and precessional light curves obtained by INTEGRAL confirms the presence of a hot extended corona in the central parts of the supercritical accretion disk and constrain the binary mass ratio in SS433 in the range $0.5 \gtrsim q \gtrsim 0.3$, confirming the black hole nature of the compact object. Orbital and precessional light curves in the hardest X-ray band 40-60 keV, which is free from emission from thermal X-ray jets, are also best fitted by the same geometrical model with hot extended corona at $q \sim 0.3$, stressing the conclusions of the modeling of the broad-band X-ray orbital and precessional light curves.
\end{abstract}

An INTEGRAL view of the high-energy sky (the first 10 years) - 9th INTEGRAL Workshop and celebration of the 10th anniversary of the - launch,

15-19 October 2012

Bibliotheque Nationale de France, Paris, France

*Speaker. 


\section{Introduction}

SS433 is a unique galactic steadily superaccreting microquasar with mildly relativistic ( $v=$ $0.26 c$ ), precessing jets located at a distance of $5.5 \mathrm{kpc}[14,2,3,7,8]$. The system exhibits three photometric and spectral periodicities related to precession $\left(P_{\text {prec }}=162^{d} .5\right)$, orbital $\left(P_{\text {orb }}=13^{d} .082\right)$ and nutation $\left(P_{\text {nut }}=6^{d} .28\right)$ periods [11]. Despite of wealth of observations, the nature of the compact star in SS433 remains inconclusive. The presence of absorption lines in the optical spectrum of the companion $[10,12]$ suggests its spectral classification as $\sim$ A7Ib supergiant. Assuming these lines to be produced in the optical star photosphere, their observed orbital Doppler shifts would correspond to the mass ratio of compact $\left(M_{x}\right)$ and optical $\left(M_{v}\right)$ star $q=M_{x} / M_{v} \sim 0.3 \pm 0.11$ and masses $M_{x}=(4.3 \pm 0.8) M_{\odot}, M_{v}=(12.3 \pm 3.3) M_{\odot}$, respectively, pointing to the black hole nature of the compact star.

Modeling of all INTEGRAL eclipses of the source available before 2010 [6] using a purely geometrical model yeilded independent constraints on the binary mass ratio $q=0.25-0.5$ with the most probable value $q=0.3$, suggesting the mass of the compact companion $M_{x} \simeq 5.3 M_{\odot}$ and the optical star $M_{v} \simeq 17.7 M_{\odot}$ for the observed optical star mass function $f_{v}=0.268 M_{\odot}$. This places SS433 among black-hole high-mass X-ray binaries. Thus SS433 can be the only known example of galactic massive $\mathrm{X}$-ray binary at an advanced evolutionary stage $[2,3]$ with supercritical accretion [16] onto a black hole [6]. Its study in different spectral bands provides invaluable information for theory of evolution of binary star and the formation of relativistic jets.

The basic picture of hard X-ray emitting regions, as emerged from analysis of X-ray data $[8,9,5,6,13]$, includes hot $\mathrm{X}$-ray jet propagating through a funnel in the supercritical accretion disk, filled with hot scattering medium (a corona). The X-ray spectrum of SS433 in the 3-100 keV range can be fitted by two-component model (thermal X-ray emission from the jet and thermal comptonization spectrum from corona) elaborated in [13]. The scattering corona parameters are: $T_{\text {cor }} \simeq 20 \mathrm{keV}$, Thomson optical depth $\tau_{T} \simeq 0.2$ and mass outflow rate in the jet $\dot{M}_{j}=3 \times 10^{19} \mathrm{~g} / \mathrm{s}$. This parameters suggest the coronal electron number density around $5 \times 10^{12} \mathrm{~cm}^{-3}$, which is typical in the wind outflowing with a velocity of $v \sim 3000 \mathrm{~km} / \mathrm{s}$ from a supercritical accretion disk with mass accretion rate onto the compact star $\dot{M} \sim 10^{-4} \mathrm{M}_{\odot} / \mathrm{yr}$ at distances $\sim 10^{12} \mathrm{~cm}$ from the center, where a Compton-thick photosphere is formed [8]. The size of the disk photosphere was independently estimated from measurements of fast optical aperiodic variability [1].

Here we analyze hard X-ray eclipses of SS433 near the T3 moment in combination with the precessional variability as observed by INTEGRAL, and interpret them in terms of our multicomponent geometrical model (see [6] for more detail).

\section{Composite X-ray light curve and jet nutation effect}

Previous dedicated INTEGRAL observations of hard X-ray eclipse are summarized in Table 1. They were all concentrated around precessional phase zero ('the T3 moment' in terms of the kinematic model of SS433 [14]), where the accretion disk is maximum opened to the observer and the X-ray flux from the source is the highest.

The composite IBIS/ISGRI 18-60 keV light curve of the primary X-ray eclipse at precessional phase zero is shown in Fig. 1 (panel 1). Data were analyzed using the IKI INTEGRAL data 
Table 1: Dedicated observations of SS433 by INTEGRAL

\begin{tabular}{lllc}
\hline Set & INTEGRAL orbits & Dates & Precessional phase $\psi_{p r}$ \\
\hline I & $67-70$ & May 2003 & $0.001-0.06$ \\
II & $555-556$ & May 2007 & $0.98-0.014$ \\
III & $608-609$ & October 2007 & $0.956-0.99$ \\
IV & $612-613$ & October 2007 & $0.030-0.064$ \\
V & $722-723$ & September 2008 & $0.057-0.091$ \\
VI & 984 & November 2010 & $0.87-0.89$ \\
& 987 & November 2010 & $0.93-0.94$ \\
VII & $1040-1041$ & April 2011 & $0.91-0.95$ \\
\hline
\end{tabular}
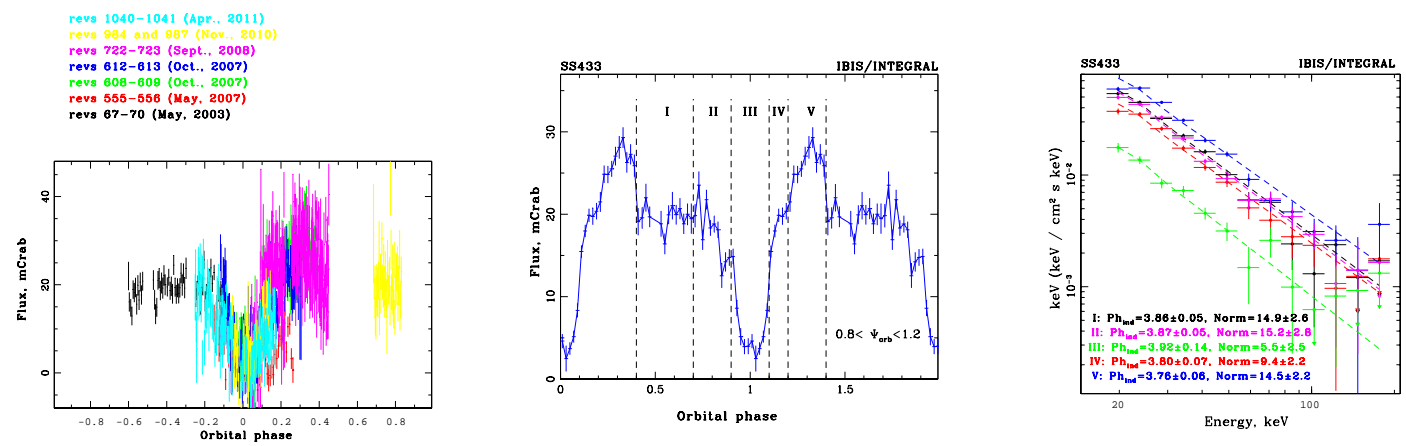

Figure 1: From left to right: 1) Composite IBIS/ISGRI 18-60 keV X-ray eclipse light curve around zero precessional phase $\psi=0.2)$ Binned $X$-ray light curve $(\Delta \phi=0.02)$ with phase intervals for spectral analysis. 3) Phase-resolved IBIS/ISGRI spectra of SS433 within chosen orbital phase intervals. 1- $\sigma$ errors are shown.

processing code described in [15]. The eclipse light curve averaged within orbital phase bins $\Delta \phi=0.02$ is shown in Fig. 1 (panel 2). $3 \sigma$-flux errors are indicated. The $18-40$ and $40-60 \mathrm{keV}$ orbital and precessional light curves with the corresponding hardness ratios are shown in Fig. 2 and Fig. 3, respectively.

A very significant flux excess at the orbital phase $\phi \sim 0.25$ is observed in the composite 18-60 light curve (Fig. 1, panel 2; Fig. 2, upper light curve) after the eclipse relative to the phase 0.75 before the eclipse. However, on the 40-60 keV light curve two maxima with similar amplitude are clearly seen at both orbital phases 0.25 and 0.75 . These $\sim 10 \%$ sine-like variability at twice the orbital period superimposed on the orbital light curve is mostly likely to be due to the jet nutation. The tidal nutation occurs twice the synodic period (6.28 $\mathrm{d}$ in the case of SS433). The effect must be maximal at the zero precessional phase when the binary system is observed in quadratures (i.e. at the binary phases 0.25 and 0.75 ). Due to nutation the jet changes its inclination to the line of sight by 6 degrees, suggesting the change in the projection area of the jet funnel $\Delta S / S \sim \Delta i_{j} / i_{j}=0.1$ (here $i_{j} \approx 59^{\circ}$ is the jet inclinatin angle at the T3 precessional phase). Thus, the composite INTEGRAL light curve allowed us to see for the first time the jet nutation in the 40-60 keV band.

2. Orbital phase-resolved spectroscopy. The accumulated data allowed us to make for the first time the orbital phase-resolved X-ray spectroscopy. Five orbital phase intervals chosen for spectral analysis are shown by the vertical dashed lines in Fig. 1 (middle panel). The obtained X-ray 


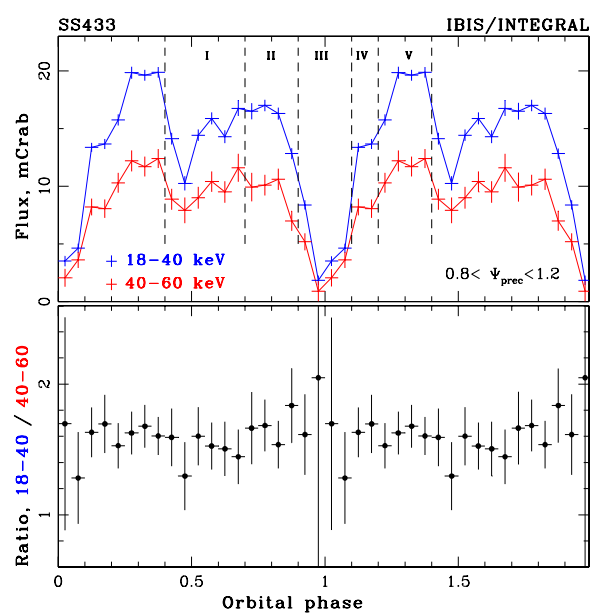

Figure 2: IBIS/ISGRI $18-40$ and $40-60 \mathrm{keV}$ orbital light curves with hardness ratio (bottom plot).

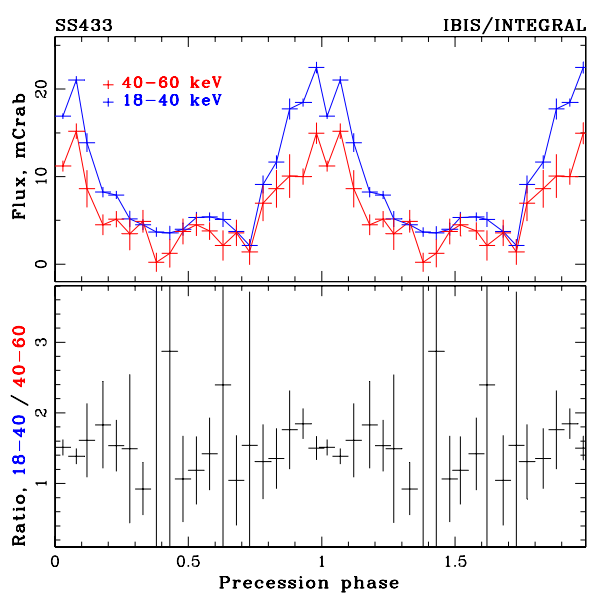

Figure 3: Precessional 18-40/40-60 keV light curves and hardness ratio.

spectra are shown in Fig. 1 (right panel). It is seen that within errors they have an identical powerlaw shape with photon spectral index $\Gamma_{p h} \simeq 3.8$. Nevertheless, a tendency of the spectrum to get harder at $\phi \sim 0.25$ (phase interval V in Fig. 1, middle panel) than at $\phi_{o r b} \sim 0.75$ (phase interval I in Fig. 1, middle panel) is clearly seen. Note also that at the middle eclipse (phase interval III in Fig. 1, middle panel) the spectrum gets softer. This is exactly what is expected in the jet nutation picture: during the disk-jet nutation the angle between the line of sight and the jet axis changes by $\sim 6$ degrees, so the observer looks into the funnel less (at $\phi_{o r b}=0.5$ ) or more (at $\phi_{o r b}=0.75$ ) deeper, thus observing cooler or hotter parts of the jet base.

3. Precessional variability. At a distance of $5.5 \mathrm{kpc}$ to SS433, reliably derived from the kinematic properties of moving emission lines [8], the observed X-ray flux 18-60 keV corresponds to a maximum uneclipsed hard X-ray luminosity of $3 \times 10^{35} \mathrm{erg} / \mathrm{s}$. The precessional change of the X-ray flux is shown in Fig. 3. To plot this Figure, all available observations of SS433 by INTEGRAL with a total exposure time of about $8 \mathrm{Ms}$ were used. This confirms the presence of a fairly broad region emitting in hard X-rays with size comparable to that of the accretion disk $\left(\sim 10^{12} \mathrm{~cm}\right)$, since (excluding orbital eclipses) the observed flux varies due to precession of the disk. The both precessional light curves show precessional variability and were used to constrain parameters of the hot corona ([6] and below).

\section{Results}

The INTEGRAL observations of SS433 provide three different light curves, which can be used to constrain the parameters of the system: 1) the orbital light curve, 2) the precessional light curve out of eclipses, and 3) precessional light curve in the middle of the eclipses (see Fig. 4, upper panels, and lower panels, blue and red crosses, respectively). We use a geometrical model described in detail in [6]. Briefly, the model includes an optical star with mass $M_{v}$ filling its Roche lobe, a compact star with mass $M_{x}$ surrounded by an optically thick accretion disk with radius $a_{d}$, 

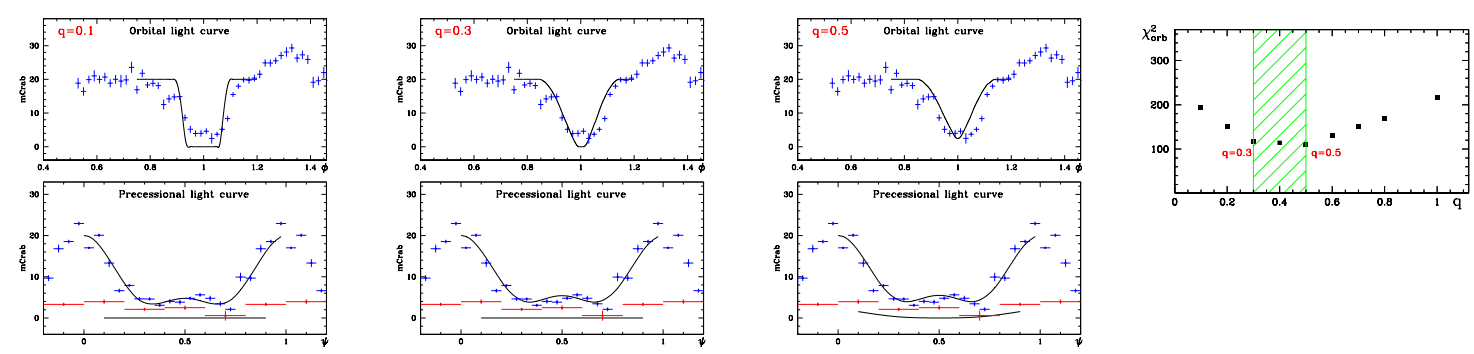

Figure 4: Joint analysis of orbital (upper plots) and precessional (lower plots) 18-60 keV light curves of SS433. On the bottom panels, precessional variability in the middle of eclipse $\left(0.95<\phi_{\text {orb }}<1.05\right)$ are shown by red crosses. 1). $q=0.1$, 'short jet' corona $\left.\left(a_{j}=0.25, b_{j}=0.1, \omega=40^{\circ}\right) .2\right) q=0.3$, 'short jet' corona $\left(a_{j}=0.35, b_{j}=0.13, \omega=80^{\circ}\right)$. 3) $q=0.3$, 'short jet' corona $\left.\left(a_{j}=0.35, b_{j}=0.13, \omega=80^{\circ}\right) .4\right)$ $\chi^{2}$ for the orbital light curve for different mass ratios.

and a hot corona which is modeled as a broad 'jet' parametrized by the part of ellipse with semiaxes $a_{j}$ and $b_{j}$ normalized to the binary orbital separation $a$. The elliptical corona is restricted by the cone with half-angle $\omega$. The shape and amplitude of the precessional light curve constrain the height of the hot X-ray corona, while the orbital light curve restricts the accretion disk radius. At a given binary mass ratio, after finding the best parameters for the precessional variability, we can calculate the deviations of the model orbital light curve from the observed one. The results of the joint analysis of the orbital and precessional 18-60 keV light curves of SS433 are shown in Fig. 4.

To avoid contamination from thermal X-ray emission from relativistic jets, we repeated the analysis using only hard X-ray 40-60 keV light curves shown in Fig. 2 and Fig. 3. The result for different mass ratios $q=M_{x} / M_{v}$ is presented in Fig. 5. It is seen that the hard X-ray orbital and precessional light curves can be simultaneously reproduced by the geometrical model for the binary mass ratio $q \gtrsim 0.3$ (at smaller mass ratios a plateau corresponding to the total eclipse of the hot corona by the optical star appears in the orbital light curve, which is not observed), in agreement with the analysis of the broadband $18-60 \mathrm{keV}$ orbital and precessional light curves shown in Fig. 4.

\section{Conclusions}

1) INTEGRAL observations of SS433 in hard X-rays $18-60 \mathrm{keV}$ allowed us for the first time to make orbital-resolved spectroscopy of the X-ray eclipse in the precessional phase corresponding to the maximum opening angle of the disk. The hard X-ray continuum is fitted with a power-law with photon index $\Gamma \approx 3.8$ which does not significantly change across the eclipse, suggesting the origin of this emission as being due to scattering in hot corona surrounding the funel around the jets in a supercritical accretion disk in SS433.

2) For the first time, joint analysis of hard $\mathrm{X}$-ray (40-60 keV) orbital and precessional light curves has been performed. This analysis independently confirms our previous result [6] that the low value of the mass ratio $q=M_{x} / M_{v}$ in SS433 cannot reproduce the observed orbital and precessional light curves. With the existing estimates of the mass function of the compact star, the most likely value $q \sim 0.3$ points to the black hole nature of the compact star in SS433. 

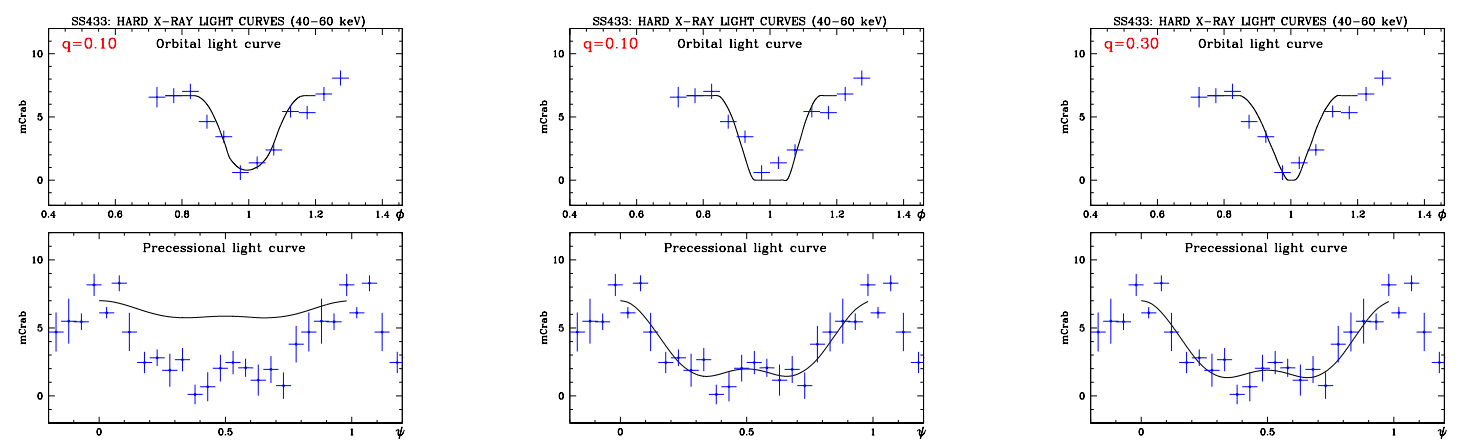

Figure 5: Joint analysis of orbital (upper plots) and precessional (lower plots) 40-60 keV light curves of SS433. Left panel: $q=0.1$, 'long jet' corona $\left(a_{j}=0.25, b_{j}=0.55, \omega=80^{\circ}\right)$; only orbital light curve can be reproduced. Middle panel: $q=0.1$, 'short jet' corona $\left(a_{j}=0.25, b_{j}=0.1, \omega=80^{\circ}\right)$. Both orbital and precessional light curvs can be fitted, but the total eclipse (plateau at zero flux) appears (unobserved). Right panel: $q=3$, 'short jet' corona $\left(a_{j}=0.35, b_{j}=0.13, \omega=80^{\circ}\right)$; both orbital and precessional light curves are well reproduced.

3) The shape of the hard X-ray orbital light curve 40-60 keV demonstrates two humps at around orbital phases 0.25 and 0.75 , likely due to the nutation effects in SS433.

New INTEGRAL observations of SS433 at different precessional phases will be used to further constrain physical parameters of this unique galactic microquasar.

\section{References}

[1] R. Burenin R. et al, Astron. Lett. 37100 (2010).

[2] A.M. Cherepashchuk, MNRAS 194761 (1981).

[3] A.M. Cherepashchuk, Sov. Sci. Rev. Ap. Space Phys. (Ed. by R.A.Sunyaev) 71 (1988).

[4] A.M. Cherepashchuk et al, AA 411441 (2003).

[5] A.M. Cherepashchuk et al, $A A 411441$ (2003).

[6] A.M. Cherepashchuk et al.,MNRAS 397479 (2009).

[7] D. Crampton, J.B. Hutchings, Vistas Astron. 2513 (1981).

[8] S.N. Fabrika, Astrophys. Space Phys. Rev. 121 (2004).

[9] E.V. Filippova et al., $A A 460125$ (2006).

[10] D.R. Gies, W. Huang, M.V. McSwain, ApJ 578 L67 (2002)

[11] V.P. Goranskij, V.F. Esipov, A.M. Cherepashchuk, Astron. Rep. 42, 209; 336 (1998).

[12] T.C. Hillwig, D.R. Gies, ApJ 676 L37 (2008).

[13] Yu. Krivosheev et al., MNRAS 3941674 (2009).

[14] B. Margon, ARAA 22507 (1984).

[15] S. Molkov et al., Astron. Lett. 30534 (2004).

[16] N.I. Shakura, R.A. Sunyaev, AA 24337 (1973). 